DOI: http://dx.doi.org/10.18509/AGB.2015.02

UDC: $551.54: 556.13$

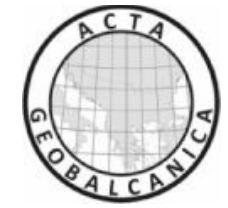

\title{
THE EFFECTS OF THE ATMOSPHERIC PRESSURE ON EVAPORATION
}

\author{
Evren Özgür ${ }^{1}$ \\ Prof. Dr. Kasım Koçak ${ }^{1}$ \\ ${ }^{1}$ Istanbul Technical University, Faculty of Aeronautics and Astronautics, \\ Department of Meteorology, Turkey \\ Corresponding author: evrenozgur87@gmail.com
}

\begin{abstract}
Evaporation is the transfer of water from liquid to gaseous state and its diffusion into the atmosphere. Evaporation from free water surface is very crucial in hydrological studies. There are lots of factors which affect evaporation. The main factors are air and water temperature, relative humidity, wind velocity, surface area, atmospheric pressure and salinity of the water. Most of the studies about evaporation are related with temperature, relative humidity and wind velocity all around the world. The other factors are usually ignored. In this study, the effect of atmospheric pressure on evaporation was examined. In this study, daily atmospheric pressure and evaporation data observed in the Ercan Meteorological Station located in Northern Cyprus were used. The observation period was taken to be 2007-2011 for both variables. By using classical scatterplot graphics, relationship between pressure and evaporation could not be observed easily. Therefore, Locally Weighted Scatterplot Smoothing (LOWESS) method was applied in order to see the net picture behind the scatterplot. At the end of the application of LOWESS, it was seen clearly that there was a distinct relationship between pressure and evaporation values. It was presented that the less evaporation was observed in high pressure values. Mathematical form of this relationship can be given in exponential form.
\end{abstract}

Keywords: Atmospheric Pressure, LOWESS, Evaporation, North Cyprus.

\section{INTRODUCTION}

Circulation of water vapor between surface and atmosphere is very crucial for all processes both in hydrology and meteorology. Water vapor is one of the most important greenhouse gases which cause climate change [1]. Evaporation is a mechanism effected by lots of factors. A part of the precipitation is hold in the air with the help of evaporation and transpiration and it returns to the atmosphere before reaching the surface. In addition, a part of the precipitation which fall into the surface evaporates toward to the atmosphere. Investigation of these losses is important especially in arid seasons [2].

Due to the reasons explained above, the relationship between evaporation and other meteorological variables has been investigated for many years. Most of the studies conducted on related literature have focused on temperature, relative humidity and wind speed. In 1992, Singh et al. examined relationship between evaporation and 
meteorological parameters in India. Wind speed, mean air temperature and solar radiation were positively correlated with evaporation. On the other hand, negative correlation was seen between relative humidity and evaporation. Relative humidity was the parameter which had highest correlation with evaporation values [3]. In the other study carried out in India, morning relative humidity and maximum temperature had a significant correlation with evaporation. The correlation coefficients were calculated as 0.95 and 0.94, respectively [4]. Another study showed that there was a strong correlation between annual relative humidity and evaporation in China in the period 1961 to 2006 [5]. On the other hand, there was not sufficient study which consider the relationship between atmospheric pressure and evaporation. It was mentioned that there was a reverse relationship between the two variables. However, it was discussed that relationship was not seen clearly [2]. In this study, detailed research was carried out about the relationship between atmospheric pressure and evaporation.

\section{DATA AND METHOD}

\subsection{Data and Study Area}

In the study, evaporation and atmospheric pressure data taken from Ercan Meteorological Station which is located in Northern Cyprus were used. The coordinates of the station is $35^{\circ} 10^{\prime} 11^{\prime \prime} \mathrm{N}$ and $33^{\circ} 38^{\prime} 28^{\prime} \mathrm{E}$. Figure 1 indicates the location of the abovementioned station.

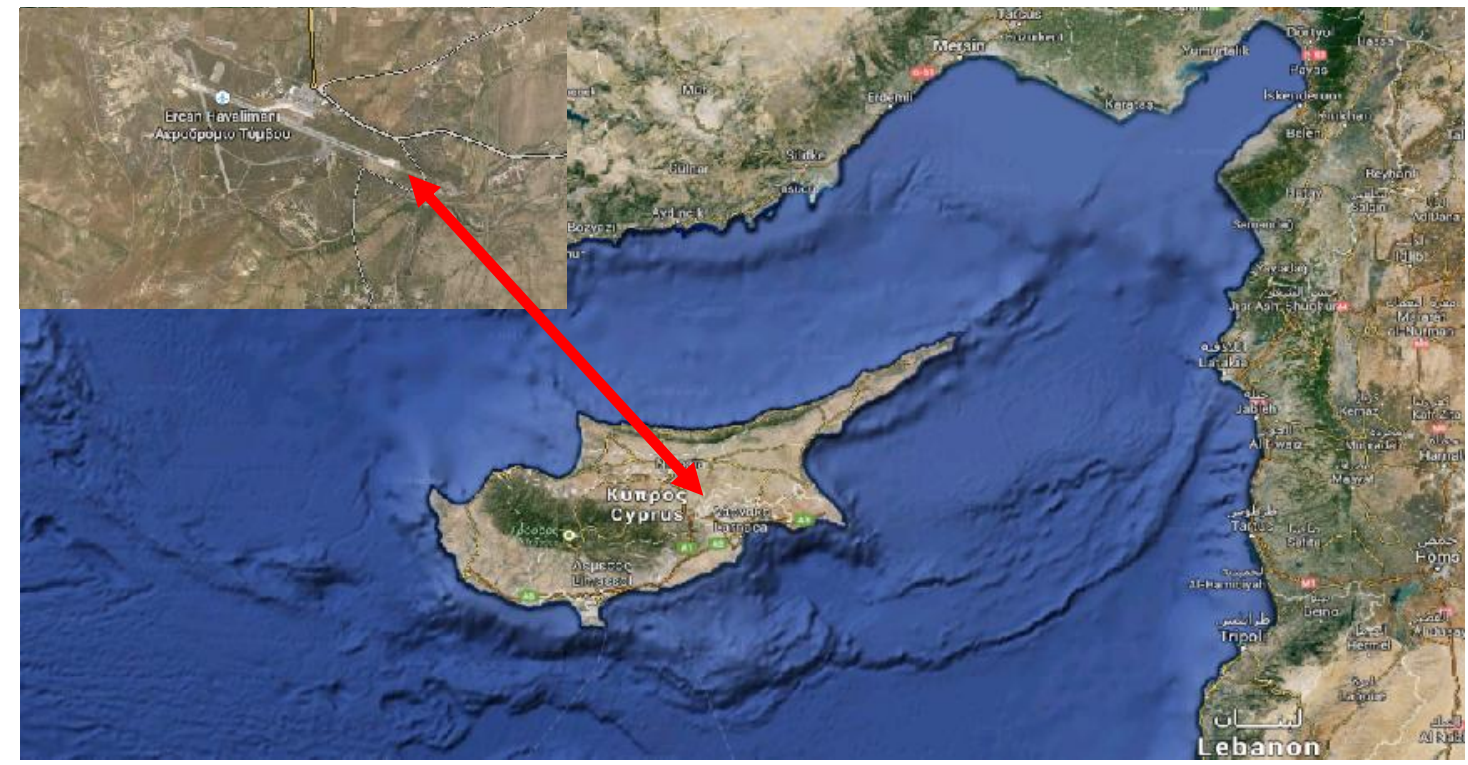

Figure 1. The location of the meteorological station.

Daily values were used for evaporation and atmospheric pressure data in the study. The units of the data were $\mathrm{mm}$ and $\mathrm{mb}$, respectively.

Figure 2 and Figure 3 show the time series of the variables used in the study. The mean and maximum values of evaporation are $6.0 \mathrm{~mm}$ and $22.5 \mathrm{~mm}$. In addition, atmospheric pressure values lie between $986.5 \mathrm{mb}$ and $1017.0 \mathrm{mb}$. The average atmospheric pressure is $998.2 \mathrm{mb}$. 


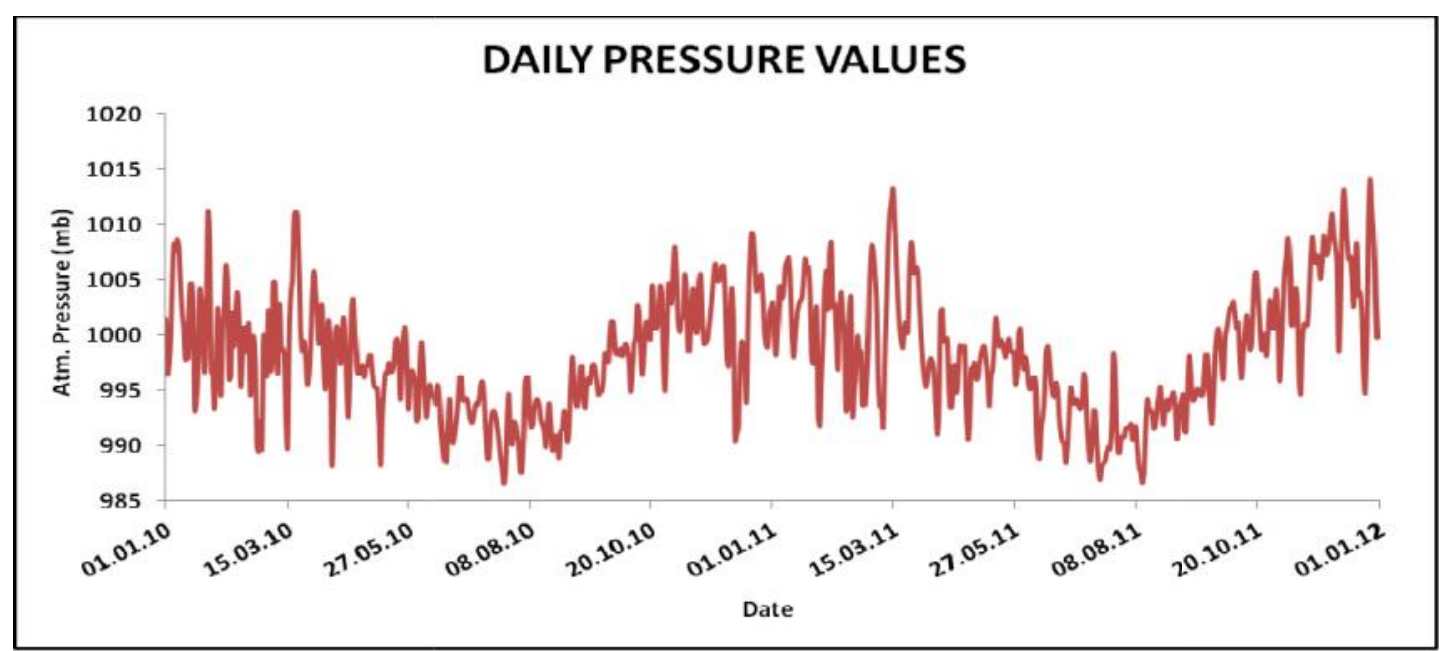

Figure 2. Time series of daily pressure values (last 2 years)

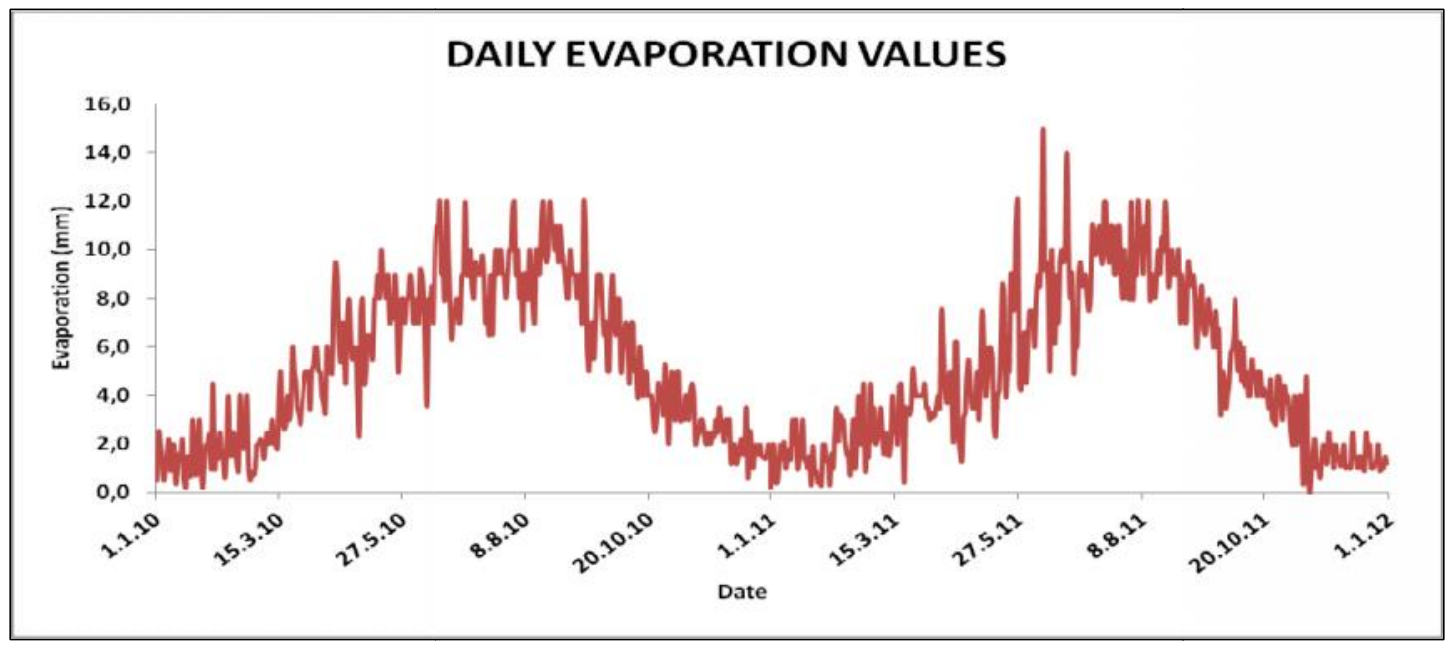

Figure 3. Time series of daily evaporation values (last 2 years)

\subsection{Method}

Smoothing is a statistical technique for removal of short term irregularities in a given time series to improve the accuracy of prediction. In other words, it is an elimination of unexpected noises from time series. There are several different smoothing techniques in scientific applications. However, most of them have serious disadvantages. Simple moving average and weighted-moving average are two of the most widely used smoothing methods all around the world. These two methods are highly vulnerable to outliers [6].

Lowess is a weighted polynomial regression method which is used to detect the relationship between two different variables. It is a procedure for fitting a regression surface to data through multivariate smoothing. The method was developed by Cleveland in 1979 and improved by Cleveland and Devlin in 1988 [7,8]. It is an outlier resistant method based on local polynomial fits. 
The locally weighted regression approach assumes that the explanatory variable $x$ and the response variable $y$ are related in the following way:

$$
y_{i}=g\left(x_{i}\right)+\varepsilon_{i}
$$

where $g$ is polynomial function in the predictor variable $x$, and $\varepsilon_{i}$ are random variables with mean 0 and constant scale. Values $y_{i}$ are used to estimate $y_{i}$ at each $x_{i j}$ and are found by fitting the polynomials using weighted least squares with large weights for points near to $x_{i}$ and small otherwise. There are two parameters that control the shape of a lowess curve. The first one is a smoothing parameter which lies between 0.25 and 1.0. If the parameter is too large, the estimate will be biased. Otherwise, if the parameter is too small, the estimate will be overfitted with inflated variance.

The second parameter is the degree of the polynomials that are fitted by the method. That parameter can be 1 or 2 . In any specific application, the change of the parameters must be based on a combination of judgement and trial and error. Residual plots may be helpful in judging a particular combination of values [9].

In order to start the smoothing procedure, the weight function must be defined. Let $W$ be a weight function with the following properties:

1. $W(x)>0$ for $|x|<1$

2. $W(-x)=W(x)$

3. $W(x)$ is a nonincreasing function for $x \geq 0$

4. $W(x)=0$ for $|x| \geq 1$

Let $0<f \leq 1$ and let $r$ be $f_{n}$ rounded to the nearest integer. For each $x_{i}, w_{k}\left(x_{i}\right)$ are defined for all $x_{i}, k=1,2, \ldots, n$ using the weight function. This is done by centering $W$ at $x_{i}$ and scaling it so that the point at which $W$ first become 0 is at the $r$ th nearest neighbor of $x_{i}$. The initial fitted value at each $x_{i}$ is fitted value of a $d$ th degree polynomial fit to the data using weighted least squares with weights $w_{k}\left(x_{i}\right)$. A different set of weights is defined for each $\left(x_{i}, y_{i}\right)$ pair based on the size of the residual $y_{i}-\hat{y}_{i}$.

Let $h_{i}$ be the distance from $x$ to the $r$ th nearest neighbor of $x_{i}$. That is, $h_{i}$ is the $r$ th smallest number among $\left|x_{i}-x_{j}\right|$ for $j=1, \ldots, n$. For $k=1, \ldots, n$,

$$
w_{k}\left(x_{i}\right)=W\left(h_{i}^{-1}\left(x_{k}-x_{i}\right)\right)
$$

Locally weighted regression and robust locally weighted regression are defined by the following operations:

1. For each estimation, $\beta_{j}\left(x_{i}\right), j=0, \ldots$ is a polynomial degree of $d$ function,

$$
\sum_{k=1}^{n} w k(x i)\left(y_{k}-\beta_{0}-\beta_{1} x_{k}-\cdots-\beta_{d} x_{k}^{d}\right)^{2}
$$

and

$$
\hat{\mathrm{y}}_{i}=\sum_{j=0}^{d} \beta_{j}\left(x_{i}\right) x_{i}^{j}=\sum_{k=1}^{n} r_{k}\left(x_{i}\right) y_{k}
$$

where $r_{k}\left(x_{i}\right)$ are the coefficients arise from the regression. 
2. Let $B$ be the weight function;

$$
\begin{gathered}
\text { for }|x|<1, B(x)=\left(1-x^{2}\right)^{2} \\
\text { for }|x| \geq 1, B(x)=0
\end{gathered}
$$

3. Compute new $\hat{y}_{i}$ for each $i$ by fitting a dth degree polynomial using weighted least squares.

4. Carry out step 2 and 3 a total of $i$ times. The final $\hat{y}_{i}$ are robust locally weighted regression fitted values. The weighted function is tricube;

$$
\begin{gathered}
\text { for }|x|<1, W(x)=\left(1-|x|^{3}\right)^{3} \\
\text { for }|x| \geq 1, W(x)=0
\end{gathered}
$$

\section{RESULTS AND DISCUSSION}

According to the classical scatterplot, the relationship between pressure and evaporation was not strong. Figure 4 and Figure 5 show the scatterplots of atmospheric pressure and evaporation.

The calculated determination coefficients $\left(\mathrm{R}^{2}\right)$ are 0.43 for linear fit and 0.46 for polynomial fit. As seen in Figure 4 and Figure 5, the relationship between atmospheric pressure and evaporation is not meaningful physically.

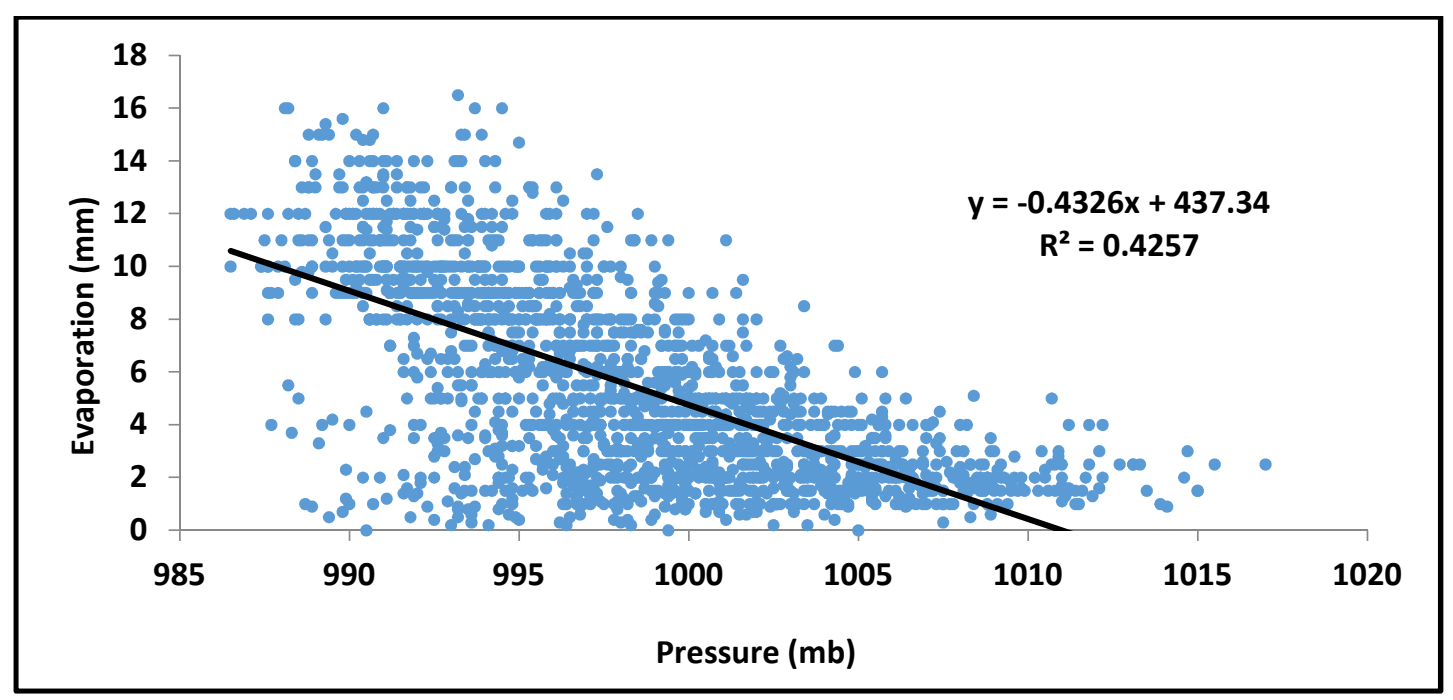

Figure 4. Scatterplot of pressure and evaporation (with linear fit)

The relationship between parameters was represented with the second degree equation. The equation was generated by using polynomial fit.

$$
\mathrm{y}=0,0173 x^{2}-34,988 x+17703
$$

where $y$ is evaporation $(\mathrm{mm})$ and $x$ is atmospheric pressure ( $\mathrm{mb})$. 


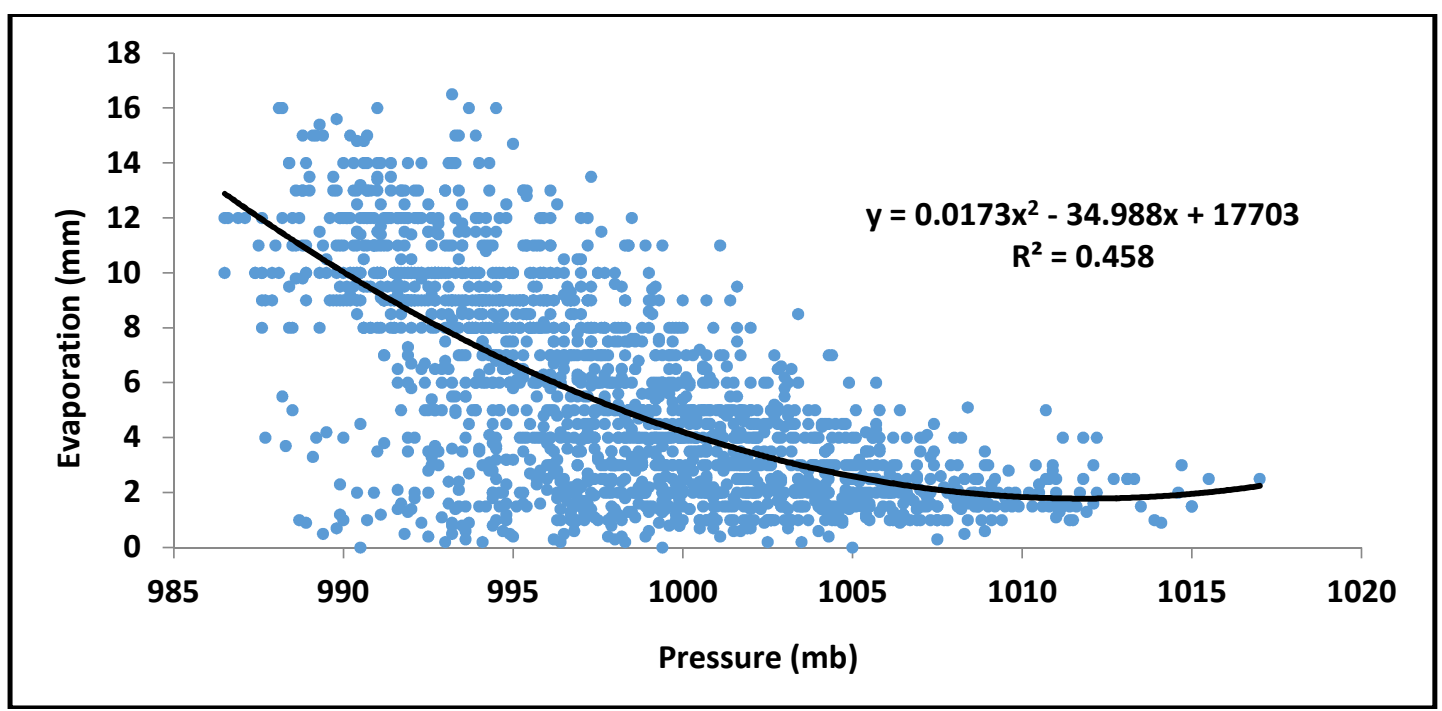

Figure 5. Scatterplot of pressure and evaporation (with polynomial fit)

After using classical scatterplot, LOWESS method was used to make the relationship more meaningful. In order to apply the method, a FORTRAN program was used. Figure 6 shows the scatterplot of both original data and smoothed data.

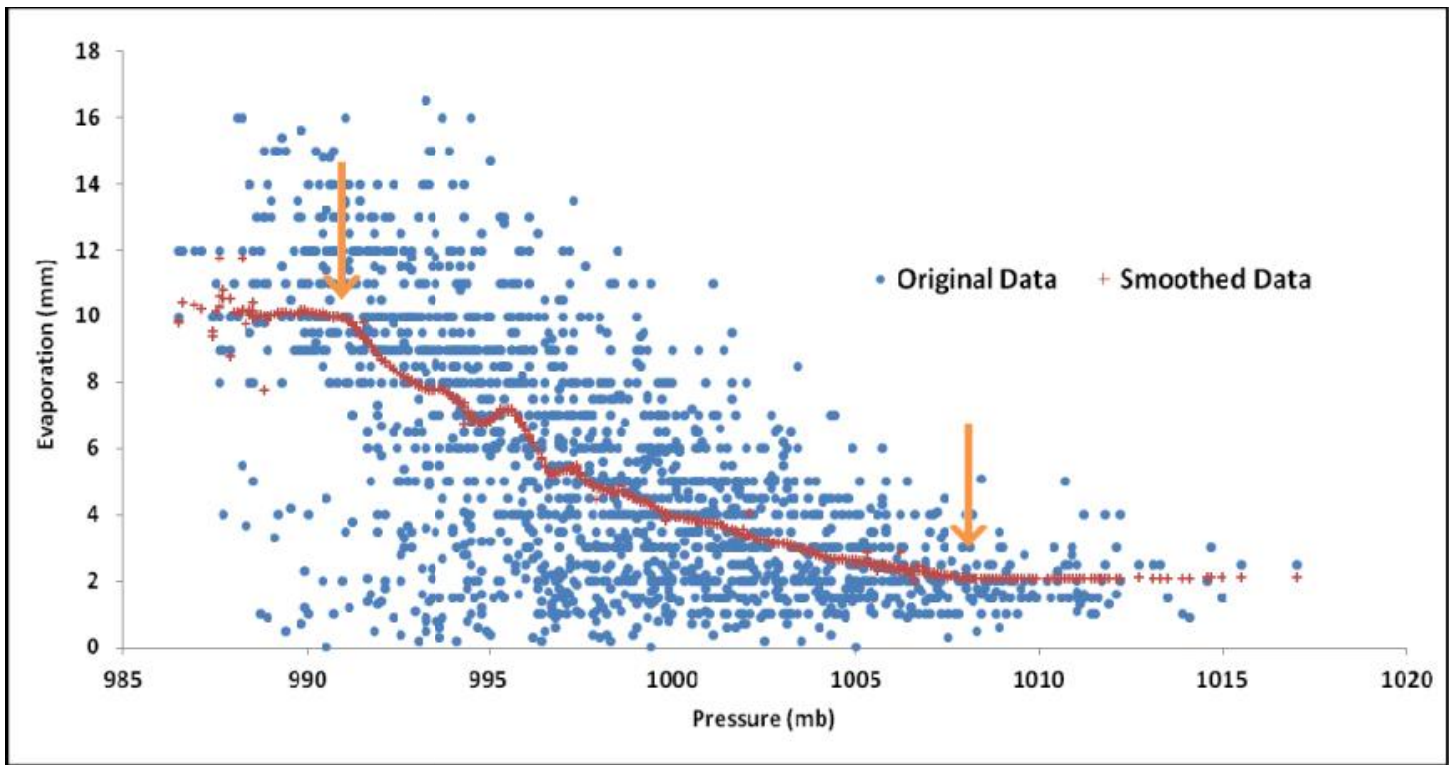

Figure 6. Scatterplot of variables (LOWESS method application)

As seen in Figure 6, three different processes have been observed. In first process, it was seen that evaporation was steady with increasing pressure until $991 \mathrm{mb}$. The constant evaporation value was $10 \mathrm{~mm}$ for this process. Second process showed that there was an exponential relationship between two variables. Decreasing evaporation was observed when pressure increase. Finally, third process indicated that evaporation stayed constant while pressure increased after $1008 \mathrm{mb}$ threshold value. The constant evaporation value after $1008 \mathrm{mb}$ was approximately $2 \mathrm{~mm}$ in third process. 
Figure 7 represents the smoothed evaporation values of second process. Detailed analyze of second process presented that smoothed values could be represented well as exponential fit. The equation of the fit was given below:

$$
y=6 * 10^{41}(\exp (-0,095 x))
$$

where $y$ is evaporation $(\mathrm{mm})$ and $x$ is atmospheric pressure (mb).

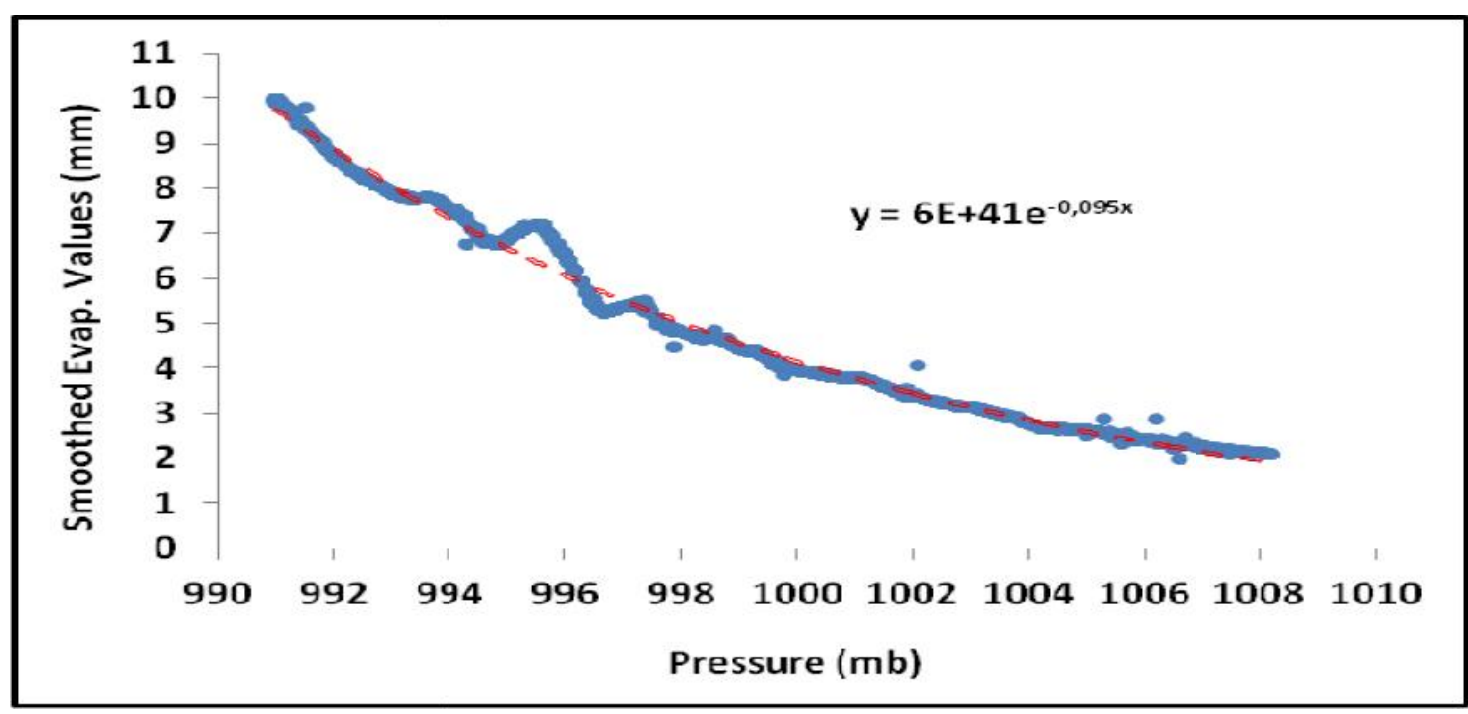

Figure 7. Smoothed evaporation values (with exponential fit)

\section{CONCLUSION}

There are lots of factors which effect evaporation. One of them is atmospheric pressure that is usually ignored. As explained before, it was attempted to make a relationship between atmospheric pressure and evaporation for Ercan Meteorological Station in the study. In order to implement this, LOWESS method was used. Remarkable results were revealed at the end of the study. Three different process was observed according to the scatterplot of LOWESS result. In lower pressure values, two different evaporation distribution was observed until $991 \mathrm{mb}$. In addition, after $1008 \mathrm{mb}$ pressure value, it was presented that evaporation did not change with increasing pressure. According to the second process, it was determined there was a reverse relationship between pressure and evaporation. It was presented that the less evaporation was observed in high pressure values. This relationship was represented with an exponantial equation. It is planned to apply the method for different stations in order to verify the results found that study. 


\section{REFERENCES}

[1] Ceyhan I, Investigation of the Relationship Between Atmospheric Pressure and Evaporation, Undergraduate Thesis, Istanbul Technical University, Turkey, 2013. (In Turkish)

[2] Bayazit M, Hydrology, Birsen Press, Istanbul, Turkey, 2003. (In Turkish)

[3] Singh R, Bishnoi O P \& Ram N, Relationship between evaporation from class 'A' open pan evaporimeter and meteorological parameters at Hisar, Haryana Agri Univ. J. Res., 22(2): 97-98, 1992.

[4] Shrivastava S K, Sahu A K, Dewangan K N, Mishra S K, Upadhyay A P \& Dubey A K, Estimating pan evaporation from meteorological data for Japalpur, Ind. J. Soil Cons., 29(3): 224-228, 2001.

[5] Yuhe J \& Guangsheng Z, Important factors governing the incompatible trends of annual pan evaporation, evidence from a small scale region, Clim. Change, 106: 303$314,2011$.

[6] Canitez N, Data Processing in Geophysics, ITU, Istanbul, Turkey, 1984. (In Turkish)

[7] Cleveland W S, Robust locally weighted regression and smoothing scatterplots, Journal of the American Statistical Association, 74: 829-836, 1979.

[8] Cleveland W S \& Devlin S J, Locally weighted regression: an approach to regression analysis by local fitting, Journal of the American Statistical Association, 83: 596-610, 1988.

[9] Everitt B S, Multivariate Modeling and Multivariate Analysis for the Behavioral Sciences, CRC Press, pp 73-74, 2009. 